\title{
Star Formation in the Early Universe
}

\author{
Kazuyuki Omukai \\ Department of Physics, Kyoto University, Kyoto, Japan \\ email: omukai@tap.scphys.kyoto-u.ac.jp
}

\begin{abstract}
In low-metallicity environments, massive stars are more easily formed than in the solar neighborhood. In this article, we see the following examples of low-mass star formation. We first describe the first star formation in the universe and argue that they are typically ordinary-sized massive stars, rather than very massive $\left(>100 M_{\odot}\right)$ ones. Next, we see how the metal-enrichment changes the thermal evolution of gas, thereby causing the shift of characteristic stellar mass towards lower mass. Finally, we discuss the possibility of forming supermassive stars in some special conditions in young galaxies.
\end{abstract}

Keywords. Star Formation, Pop II Stars, Pop III Stars

\section{Metallicity and Massive Star Formation}

Stars are formed as a result of gravitational collapse of dense molecular cores and subsequent accretion of ambient matter. In the standard picture of low-mass star formation, the accretion rate is approximately given by dividing the Jeans mass, $M_{\text {Jeans }}$, by the free-fall time $t_{\mathrm{ff}}: \dot{M}_{*} \sim M_{\mathrm{Jeans}} / t_{\mathrm{ff}} \simeq c_{\mathrm{s}}^{3} / G \simeq 2 \times 10^{-6} M_{\odot} / \mathrm{yr}\left(\frac{T}{10 \mathrm{~K}}\right)^{3 / 2}$, where $c_{\mathrm{s}}$ is the sound speed in the dense core.

In trying to apply the same formation scenario to massive stars ( $>$ a few $10 \mathrm{~s} M_{\odot}$ ) just with prolonged accretion, however, we meet the following difficulties. First, with accretion rates as low as $10^{-6} M_{\odot} / \mathrm{yr}$, the time needed to form a massive star (with mass $\left.M_{*}\right) t_{\mathrm{acc}}=M_{*} / \dot{M}_{*}$ exceeds the stellar lifetime $t_{\mathrm{OB}} \simeq 3 \times 10^{6} \mathrm{yr}$. Second, the radiation pressure onto the dust grains in the accretion flow becomes stronger than the ram pressure of the flow, thereby halting the accretion (e.g., Wolfire \& Cassinelli 1987).

In low-metallicity (and thus low dust-depletion) gas, due to inefficient cooling, clouds are warmer than the present-day counterparts, which results in the higher accretion rate. In addition, the reduced dust opacity means lower radiation pressure. Those effects mitigate the above mentioned obstacles in massive star formation. We thus naturally expect that massive stars are more abundant in low-metallicity environments.

In the following, we see specific examples of low-metallicity star formation. In Sec. 2, we describe the first star formation in the universe and argue that they are typically ordinarysized massive stars, rather than very massive $\left(>100 M_{\odot}\right)$ ones as previously considered. Next, in Sec. 3, we see how the metal-enrichment changes the thermal evolution of gas, thereby causing a shift of the characteristic stellar mass towards lower mass. Finally, in Sec. 4, we discuss the possibility of forming supermassive stars (SMSs) in some special conditions in young galaxies.

\section{First Stars: Massive but Not Very Massive}

According to our current understanding of star formation, the dense core of a gas cloud gravitationally contracts in a non-homologous run-away fashion, whereby the densest parts become denser faster than the rest of the cloud. In a primordial gas cloud one or 
a few embryo protostars are formed near the center. The initial mass of these embryo protostars is only $\simeq 0.01 M_{\odot}$; the bulk of the dense core material remains in the surrounding envelope and is subsequently drawn toward the protostar(s) through gravity. With the typical angular momentum of dense cores, the centrifugal barrier allows only a small amount of infalling gas to accrete directly onto the star. Instead, a circumstellar disk is formed and the gas is accreted onto the central star through the disk. The final mass of these first stars is fixed, when the mass accretion terminates.

Here, we report the calculation by Hosokawa et al. (2011). We follow the radiation hydrodynamic evolution in the vicinity of an accreting protostar, incorporating thermal and chemical processes in the primordial gas in a direct manner. We also follow the evolution of the central protostar self-consistently by solving the detailed structure of the stellar interior with zero metallicity as well as the accretion flow near the stellar surface. We configure the initial conditions by using the results of a three-dimensional cosmological simulation, which followed the entire history from primeval density fluctuations to the birth of a small "seed" protostar at the cosmological redshift 14. Specifically, when the maximum particle number density reaches $10^{6} \mathrm{~cm}^{-3}$ in the cosmological simulation, we consider a gravitationally bound sphere of radius $0.3 \mathrm{pc}$ around the density peak, which encloses a total gas mass of $\simeq 300 M_{\odot}$. We reduce the three-dimensional data to an axisymmetric structure by averaging over azimuthal angles.

At its birth a very small protostar of $\sim 0.01 M_{\odot}$ is surrounded by a molecular gas envelope of $\sim 1 M_{\odot}$, which is quickly accreted onto the protostar. Atomic gas further out initially has too much angular momentum to be accreted directly and a circumstellar disk forms. The infalling atomic gas first hits the disk plane roughly vertically at supersonic velocities. A shock front forms; behind the shock the gas cools, settles onto the disk, and its hydrogen is converted to the molecular form via rapid gas phase three-body reactions. The molecular disk extends out to $\simeq 400 \mathrm{AU}$ from the protostar, when the stellar mass is $10 M_{\odot}$. Accretion onto the protostar proceeds through this molecular disk as angular momentum is transported outward. The accretion rate onto the protostar is $\simeq 1.6 \times 10^{-3} M_{\odot} \mathrm{yr}^{-1}$ at this moment.

Fig. 1 depicts the highly dynamical behavior of the circumstellar material in the late $\mathrm{KH}$ contraction phase. When the stellar mass is $20 M_{\odot}$, an ionized region rapidly expands in a bipolar shape perpendicular to the disk, where gas is cleared away (Fig. 1-a). At this moment the disk extends out to $\simeq 600 \mathrm{AU}$. The disk is self-shielded against the stellar $\mathrm{H}_{2}$-dissociating $(11.2 \mathrm{eV} \leqslant h \nu \leqslant 13.6 \mathrm{eV})$ as well as the ionizing radiation. The Hil region continues to grow and finally breaks out of the accreting envelope. At $M_{*} \simeq 25 M_{\odot}$, the size of the bipolar HiI region exceeds 0.1 pc (Fig. 1-b). Because of the high pressure of the heated ionized gas, the opening angle of the ionized region also increases as the star grows (Fig. 1-c). Shocks propagate into the envelope preceding the expansion of the ionized region. The shocked gas is accelerated outward at a velocity of several kilometers per second. The shock even reaches regions shielded against direct stellar UV irradiation. The outflowing gas stops the infall of material from the envelope onto the disk. Without the replenishment of disk material from the envelope the accretion rate onto the protostar decreases (Fig. 2). In addition, the absence of accreting material onto the circumstellar disk means that the disk is exposed to the intense ionizing radiation from the star. The resulting photoevaporation of disk gas also reduces the accretion rate onto the protostar. The photoevaporated gas escapes toward the polar direction within the ionized region. The typical velocity of the flow is several tens of kilometers per second, comparable to the sound speed of the ionized gas, which is high enough for the evaporating flow to escape from the gravitational potential well of the dark matter halo. 


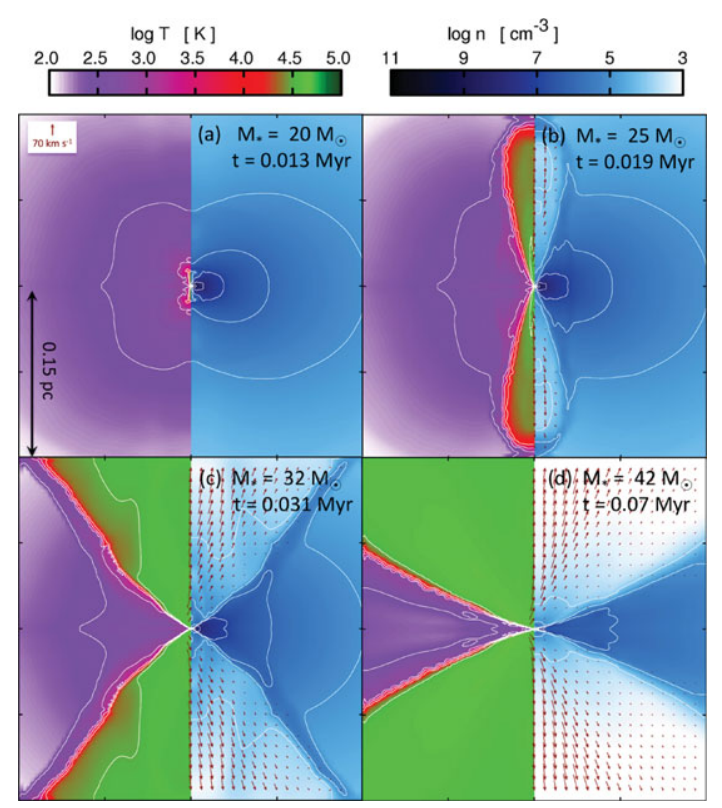

Figure 1. UV radiative feedback from the primordial protostar. The spatial distributions of gas temperature (left), number density (right), and velocity (right, arrows) are presented in each panel for the central regions of the computational domain. The four panels show snapshots at times, when the stellar mass is $M_{*}=20 M_{\odot}($ panel $a), 25 M_{\odot}(b), 35 M_{\odot}(c)$, and $42 M_{\odot}(d)$. The elapsed time since the birth of the primordial protostar is labeled in each panel. Figure from Hosokawa et al. (2011)

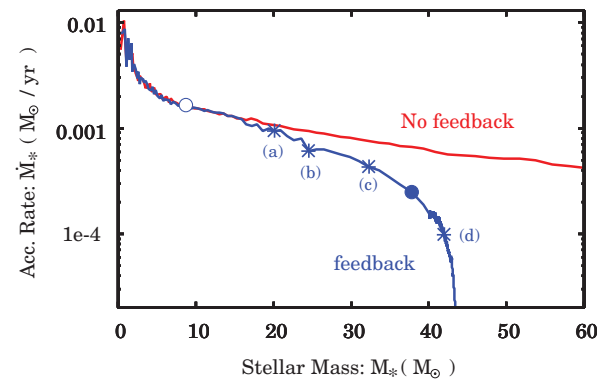

Figure 2. Evolution of the accretion rate onto the primordial protostar. The blue line represents the evolution, which includes the effect of UV radiative feedback from the protostar. The red line depicts a numerical experiment with no UV feedback. The open and filled circles denote the characteristic epochs of the protostellar evolution, beginning of the $\mathrm{KH}$ contraction and the protostar's arrival to the ZAMS. The panels (a) - (d) in Figure 1 show the snapshots at the moments marked with the asterisks. Figure from Hosokawa et al. (2011)

When central nuclear hydrogen burning first commences at a stellar mass of $35 M_{\odot}$, it is via the pp-chain normally associated with low mass stars. The primordial material does not have the nuclear catalysts necessary for CNO-cycle hydrogen burning. Because the pp-chain alone cannot produce nuclear energy at the rate necessary to cover the radiative energy loss from the stellar surface, the star continues to contract until central temperatures and densities attain values that enable the $3-\alpha$ process (helium burning). The product of helium burning is carbon and once the relative mass abundance of carbon reaches $\sim 10^{-12}$, CNO-cycle hydrogen burning takes over as the principal source of nuclear energy production, albeit at much higher central densities and temperatures than 


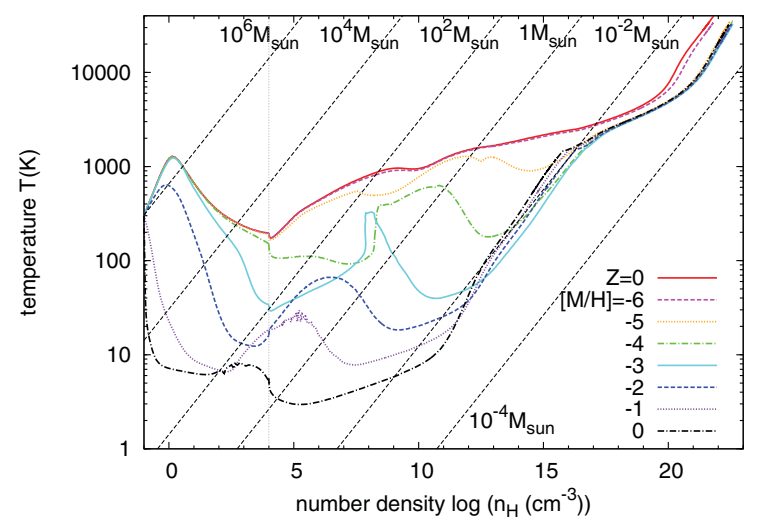

Figure 3. Temperature evolution at the center of pre-stellar clouds with different metallicities. Those with metallicities $[\mathrm{M} / \mathrm{H}]=-\infty(\mathrm{Z}=0),-6,-5,-4,-3,-2,-1$ and 0 are shown. No external radiation is imposed. The lines for constant Jeans mass are indicated by thin dotted lines. The thermal evolution is computed by a one-zone model up to the number density of $10^{4} \mathrm{~cm}^{-3}$ (dashed horizontal line), and by a hydrodynamical model for higher density. Figure from Omukai et al. (2010)

stars with solar abundances. These first generation ZAMS (zero age main sequence) stars are thus more compact and hotter than their present day counterparts of equal mass. By the time the star attains $40 M_{\odot}$, the entire region above and below the disk (Fig. 1-d) is ionized. Mass accretion is terminated when the stellar mass is $45 M_{\odot}$ (Fig. 2).

Our calculation shows that the first stars regulate their growth by their own radiation. The first stars are not extremely massive, but rather similar in mass to the O-type stars in our Galaxy. This resolves a long-standing enigma regarding the elemental abundance patterns of the Galactic oldest metal-poor stars, which contain atmospheric signatures from the earliest generation of stars. If a significant number of first stars had masses in excess of $100 M_{\odot}$, they would end their lives through pair-instability supernovae, expelling heavy elements that would imprint a characteristic nucleosynthetic signature to the elemental abundances in metal-poor stars. However, no such signatures have been detected in the metal-poor stars in the Galactic halo. For example, metals released by pair-instability supernovae are characterized by a small relative abundance of $\mathrm{Zn}$ to Fe, in contrast to observations. Detailed spectroscopic studies of extremely metal-deficient stars rather suggest that the metal-poor stars were born in an interstellar medium that had been metal-enriched by supernovae with ordinary massive stellar progenitors. If first stars have masses in the range of several tens $M_{\odot}$, as predicted by our calculations, and ultimately explode as core-collapse supernovae, the associated nucleosynthetic signatures are consistent with those observed in hyper-metal-poor stars.

\section{Effect of Metal-Enrichment: Pop III/II Transition}

The first stars in the universe were massive, typically $\sim$ a few $10 \mathrm{~s} M_{\odot}$. Stars in the solar neighborhood, on the other hand, are typically low-mass objects: the initial mass function exhibits a peak around $0.1-1 M_{\odot}$. The origin of these characteristic masses can be attributed to the thermal evolution of pre-stellar clouds (e.g., Larson 2005).

In Figure 3, we show the temperature evolution of star-forming cores with different metallicities (Omukai et al. 2010). This is calculated by using a radiation hydrodynamical calculation assuming spherical symmetry. The initial density of the hydrodynamical calculation is taken at $10^{4} \mathrm{~cm}^{-3}$, until which, we followed the evolution by a one-zone 
model. Here, the dust to metal ratio is assumed the same as the local ISM value. The dotted lines indicate the constant Jeans mass.

The temperature of metal-free gas has a minimum around $10^{4} \mathrm{~cm}^{-3}$, where the rotational levels of $\mathrm{H}_{2}$ reach LTE and its cooling rate saturates. Up to this point, the rapid cooling allows the temperature to decrease with increasing density, i.e., the effective ratio of specific heat $\gamma=d \log p / d \log \rho<1$. In such a condition, clouds in shapes other than the sphere, e.g., filaments, which eventually fragment into smaller pieces, can also collapse gravitationally. However, once the temperature begins to increase with density, filamentary clouds are no longer able to collapse due to increasing pressure support, and only spherical clouds can collapse thereafter. As a consequence, spherical clouds are formed at this moment. Since spherical clouds are hard to fragment, the typical epoch of fragmentation is around this temperature minimum, and the Jeans mass there is imprinted as the characteristic fragmentation mass scale. This has been confirmed by numerical simulations adopting turbulent initial conditions (Li et al. 2003; Jappsen et al. 2005). In the case of zero-metallicity gas, this characteristic fragmentation mass is given by the Jeans mass around $10^{4} \mathrm{~cm}^{-3}$ and is about $1000 M_{\odot}$.

On the other hand, the solar-metallicity gas has a temperature minimum at $\sim 10^{5} \mathrm{~cm}^{-3}$. This is due to the cooling by infrared emission of the dust, which makes the temperature decreases until the thermal coupling of the gas and dust is reached. The Jeans mass at this temperature minimum is $\sim M_{\odot}$, which agrees with the observed characteristic mass in the solar neighborhood.

How about the slightly metal-enriched clouds? Below the metallicity $[\mathrm{M} / \mathrm{H}]=-6$, the temperature evolution is the same as that of zero-metallicity. With metallicity of $[\mathrm{M} / \mathrm{H}] \sim-(4-5)$, the evolutionary track of the temperature has two minima: the first one at lower density is due to line-emission cooling, i.e., $\mathrm{H}_{2}$ and $\mathrm{HD}$ cooling at low $([\mathrm{M} / \mathrm{H}]<\sim-3.5)$ metallicity, and to $\mathrm{C}$ and $\mathrm{O}$ fine-structure line cooling at higher metallicity. The second temperature minimum at higher density is due to dust cooling. The existence of the two temperature minima corresponds to two fragmentation epochs. Note that the fragmentation mass scales, which are given by the Jeans masses at the temperature minima, depend only moderately on the metallicity, being very large, 100$1000 M_{\odot}$ when associated to line-cooling, and low, $0.1-1 M_{\odot}$, when associated to dustcooling. It is only dust-cooling that enables low-mass fragmentation, which implies that dust is indispensable for the formation of low-mass stars in low-metallicity gas.

Then, how much metallicity, or correctly speaking, the amount of dust, is needed for causing fragmentation and thus producing low-mass cores? This threshold value is often called the critical metallicity in literature (e.g., Schneider et al. 2002). To pin down this value, we have studied the evolution of pre-stellar cores in the dust-cooling phase by SPH simulations (Tsuribe \& Omukai 2006). We assumed that the cores are initially moderately elongated and have some random velocity perturbations. We found that (i) important quantities for telling whether the cores fragment are the elongation of cores defined by (axis ratio) - 1, and the elongation factor, that is, how many times the elongation grows from the initial value at the onset of dust-cooling phase; (ii) if the elongation is order of unity at the onset of dust-cooling phase and the elongation factor calculated by the linear theory exceeds $\simeq 3$, the core becomes very elongated with the actual elongation factor $\sim 30$ and finally fragments into many pieces during the dust-cooling phase. By applying this criterion to the thermal evolution presented in Figure 3, we find that as long as a core is moderately elongated at the beginning of the dust cooling, it will fragment for $[\mathrm{M} / \mathrm{H}]>-5$.

Thus, as a rule of thumb, we may say that the critical metallicity is $[\mathrm{M} / \mathrm{H}]_{\mathrm{cr}} \simeq-5$, although the exact value depends on the dust model we assume and may change by as 
much as an order of magnitude (e.g., Schneider et al. 2006). In any case, we conclude that only with slight metal enrichment, low-mass fragments can be produced as long as a sizable fraction of metals have condensed in the dust.

\section{Possibility of Supermassive Star Formation}

In this section, we consider the possibility of SMS formation in a rare environment in first galaxies. If formed, these can be the seeds of supermassive black holes that exist already when the age of the universe is $\lesssim 1$ Gyr (e.g., Mortlock et al. 2011).

The possibility of massive seed BH formation by direct collapse of SMS $\left(\gtrsim 10^{5} \mathrm{M}_{\odot}\right)$ has been considered by some authors. Specifically, SMS formation in massive halos $\left(T_{\mathrm{vir}} \gtrsim\right.$ $10^{4} \mathrm{~K}$ ) irradiated with strong far ultraviolet (FUV) radiation has often been studied (e.g., Shang, Bryan \& Haiman 2010). Since the $\mathrm{H}_{2}$ molecule, the main coolant in the primordial gas, is photo-dissociated with strong FUV radiation in the Lyman and Werner bands, clouds under such an environment collapse isothermally at $\sim 8000 \mathrm{~K}$ by Ly $\alpha$ cooling without fragmentation, if they are massive enough $\left(\gtrsim 10^{5} \mathrm{M}_{\odot}\right)$. As an outcome of such collapse, SMSs are expected to form. A massive seed BH as a remnant of SMS collapse reduces the growth time to $10^{9} \mathrm{M}_{\odot}$ within $0.46 \mathrm{Gyr}$ and mitigates the growthtime problem by a big margin. This scenario, however, has a serious drawback: for this mechanism of SMS formation to work, extremely strong FUV radiation $J_{21}^{\mathrm{LW}} \gtrsim 10^{2}-10^{3}$ (in units of $10^{-21} \mathrm{erg} \mathrm{s}^{-1} \mathrm{~cm}^{-2} \mathrm{~Hz}^{-1} \mathrm{sr}^{-1}$ ) is required (Omukai 2001; Shang, Bryan \& Haiman 2010), while the fraction of halos irradiated with such intense FUV fields with $J_{21}^{\mathrm{LW}} \gtrsim 10^{3}$ is estimated to be $\lesssim 10^{-6}$ at $z \sim 10$ (Dijkstra et al. 2008), i.e., only extremely rare halos satisfy the condition for SMS formation. Although the above scenario might be still viable considering the rarity of high-z SMBHs, it is worthwhile to explore another possibility.

Here, we propose a new scenario for SMS formation in post-shock gas of cold accretion flows in forming first galaxies. Recent numerical simulations of galaxy formation have revealed that, in halos with virial temperature $T_{\text {vir }} \gtrsim 10^{4} \mathrm{~K}$, the accreting cold gas penetrates deep to the center through dense filamentary flows (e.g., Dekel \& Birnboim 2006). The supersonic flows collide with each other and the resultant shock develops a hot and dense $\left(\sim 10^{4} \mathrm{~K}\right.$ and $\left.\sim 10^{3} \mathrm{~cm}^{-3}\right)$ gas near the center. By studying thermal evolution of the shocked gas, we find that, if the post-shock density is high enough for the $\mathrm{H}_{2}$ vibrational levels to reach the local thermodynamic equilibrium (LTE), the efficient collisional dissociation suppresses $\mathrm{H}_{2}$ cooling, and the gas cannot cool below several thousand $\mathrm{K}$ (Figure 4 ). Massive clouds with $\gtrsim 10^{5} \mathrm{M}_{\odot}$ formed by fragmentation of the post-shock layer subsequently collapse isothermally at $\sim 8000 \mathrm{~K}$ by the Ly $\alpha$ cooling. Without further fragmentation, monolithic collapse of the clouds results in the SMS formation. Note that, unlike the previous SMS formation mechanism, strong FUV radiation is not required in our scenario.

The mass of protostars formed via the isothermal collapse is initially only $\sim 10^{-2} M_{\odot}$ but quickly increases via mass accretion. The expected accretion rates are $0.1-1 M_{\odot} \mathrm{yr}^{-1}$, more than 100 times higher than the standard value $\simeq 10^{-3} M_{\odot} \mathrm{yr}^{-1}$ expected for Pop III star formation. The stellar mass could reach $10^{5-6} M_{\odot}$ in $\sim 1 \mathrm{Myr}$ with this very rapid mass accretion. General relativity predicts that such a supermassive star (SMS) becomes unstable and collapses to form a $\mathrm{BH}$, which subsequently swallows most of the surrounding stellar material.

We present here the evolution of protostars under such extreme conditions of rapid mass accretion (Fig. 4). Our results show that rapid accretion with $\dot{M}_{*} \gtrsim 10^{-2} M_{\odot} \mathrm{yr}^{-1}$ causes the star to bloat up like a red giant. The stellar radius increases monotonically 


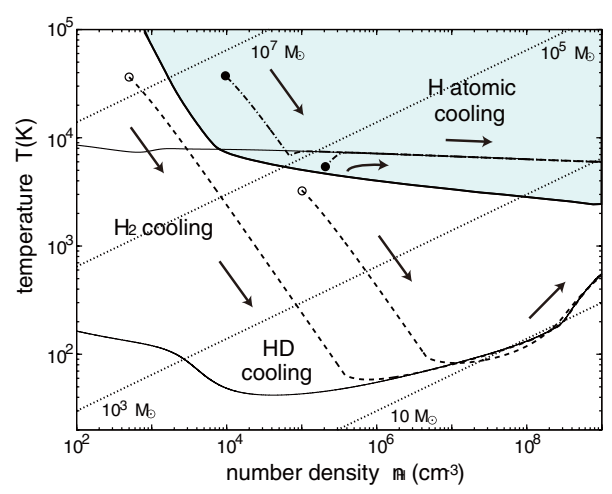

Figure 4. The temperature evolution of primordial gas after heating by the cold accretion shock for the initial ionization degree $x_{\mathrm{e}, 0}=10^{-2}$ and $\mathrm{H}_{2}$ fraction $x_{\mathrm{H}_{2}, 0}=10^{-6}$. The evolutionary tracks are shown with dashed and dash-dotted lines for four combinations of initial temperature and density, each indicated by two open and filled circles. From low-density or low-temperature initial conditions (dashed lines starting from the open circles), the temperature decreases below $\sim 100 \mathrm{~K}$ owing to the $\mathrm{H}_{2}$ and $\mathrm{HD}$ cooling. On the other hand, from dense and hot initial conditions (dash-dotted lines from the filled circles), the clouds do not cool below $\sim 8000 \mathrm{~K}$ and subsequently collapse almost isothermally by the $\mathrm{H}$ atomic cooling. The triangle symbol on each track indicates the epoch when the post-shock layer fragments by the gravitational instability. The thick solid line, the domain above which is hatched, divides the initial conditions leading to these two different ways of thermal evolution. The two thin solid lines show the temperature evolution by the $\mathrm{H}$ atomic cooling (upper) and the $\mathrm{H}_{2}$ and $\mathrm{HD}$ cooling (lower), respectively. The diagonal dotted lines (lower-left to upper-right) indicate the constant Jeans masses, whose values are denoted by numbers in the Figure. Figure from Inayoshi \& Omukai (2012)

with stellar mass and reaches $\simeq 7000 R_{\odot}(\simeq 30 \mathrm{AU})$ at a mass of $10^{3} M_{\odot}$. Unlike the cases with lower accretion rates previously studied, the mass-radius relation in this phase is almost independent of the assumed accretion rate. In addition, the effective temperature of those bloated stars is almost constant at $T_{\text {eff }} \simeq 5000 \mathrm{~K}$ due to the strong temperaturedependence of $\mathrm{H}^{-}$absorption opacity.

We have calculated the early evolution until the stellar mass reaches $10^{3} M_{\odot}$. The subsequent evolution remains unexplored because of convergence difficulties with the current numerical codes. If the star continues to expand following the same mass-radius relation also for $M_{*}>10^{3} M_{\odot}$, the stellar radius at $10^{5} M_{\odot}$ would be $\simeq 400 \mathrm{AU}$. Since the stellar effective temperature remains $\simeq 5000 \mathrm{~K}$, the star hardly emits ionizing photons during accretion. Therefore, it is unlikely that stellar growth is limited by the radiative feedback via formation of an HII region as discussed in Section 2. Such massive "super-giant" protostars could be the progenitors that eventually evolve to the observed SMBHs in the early universe.

\section{References}

Dekel, A. \& Birnboim, Y. 2006, MNRAS, 368, 2

Dijkstra, M., Haiman, Z., Mesinger, A., \& Wyithe, J. S. B. 2008, MNRAS, 391, 1961

Hosokawa, T., Omukai, K., Yoshida, N., \& Yorke, H. W. 2011, Science, 334, 1250

Hosokawa, T., Omukai, K., \& Yorke, H. W. 2012, ArXiv:1203.2613

Inayoshi, K. \& Omukai, K. 2012, MNRAS, in press (arXiv:1202.5380)

Jappsen, A.-K., Klessen, R. S., Larson, R. B., Li, Y., \& Mac Low, M.-M. 2005, A\& A, 435, 611

Larson, R. B. 2005, MNRAS, 359, 21

Li, Y., Klessen, R. S., \& Mc Low, M.-M. 2003, ApJ, 592, 975

Mortlock, D. J. et al. 2011, Nature, 474, 616 


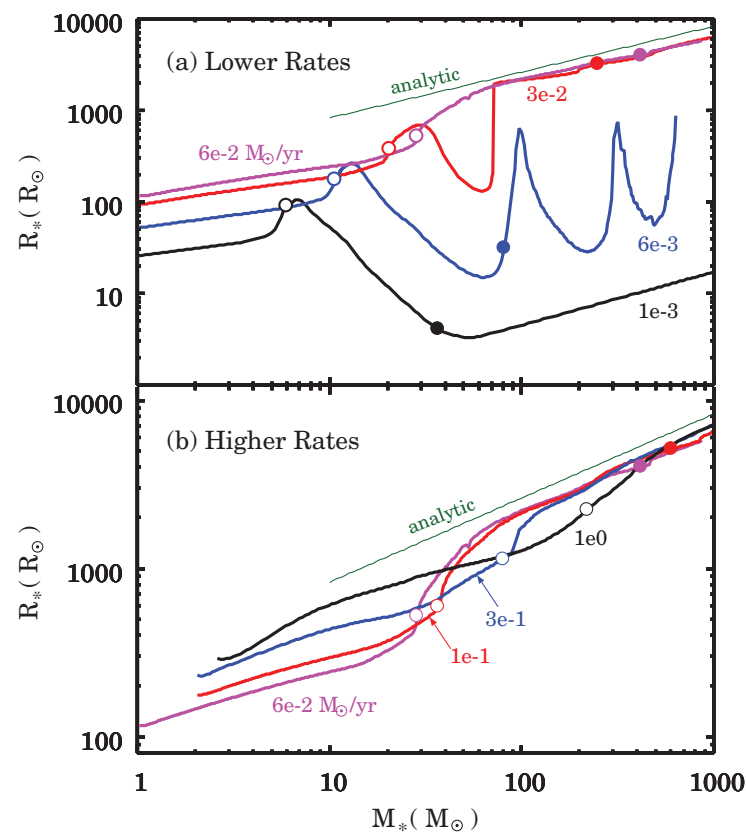

Figure 5. Evolution of the protostellar radius for various accretion rates. Upper panel: the different curves represent the cases with $\dot{M}_{*}=10^{-3} M_{\odot} \mathrm{yr}^{-1}, 6 \times 10^{-3} M_{\odot} \mathrm{yr}^{-1}, 3 \times 10^{-2} M_{\odot} \mathrm{yr}^{-1}$, and $6 \times 10^{-2} M_{\odot} \mathrm{yr}^{-1}$. The open and filled circles on each curve denote the epoch when $t_{\mathrm{KH}}=t_{\mathrm{acc}}$ and when the central hydrogen burning begins, respectively. Lower panel: same as the upper panel but for higher accretion rates of $1 \times 10^{-2} M_{\odot} \mathrm{yr}^{-1}, 0.1 M_{\odot} \mathrm{yr}^{-1}, 0.3 M_{\odot} \mathrm{yr}^{-1}$, and $1 M_{\odot} \mathrm{yr}^{-1}$. The case with $6 \times 10^{-2} M_{\odot} \mathrm{yr}^{-1}$ is illustrated in both panels as a reference. For the cases with $0.3 M_{\odot} \mathrm{yr}^{-1}$ and $1 M_{\odot} \mathrm{yr}^{-1}$ hydrogen fusion has not ignited by the time the stellar mass reaches $10^{3} M_{\odot}$. In the both panels the thin green line represents the mass-radius relation given by an analytic expression. Figure from Hosokawa, Omukai, \& Yorke (2012)

Omukai, K. 2001, ApJ, 546, 635

Omukai, K., Hosokawa, T., \& Yoshida, N. 2010, ApJ, 722, 1793

Schneider, R., Ferrara, A., Natarajan, P., \& Omukai, K. 2002, ApJ 571, 30

Schneider, R., Omukai, K., Inoue, A. K., \& Ferrara, A. 2006, MNRAS, 369, 1437

Shang, C., Bryan, G. L., \& Haiman, Z. 2010, MNRAS, 402, 1249

Tsuribe, T. \& Omukai, K. 2006, ApJ, 642, L61

Wolfire M. G. \& Cassinelli, J. P. 1987, ApJ, 319, 850

\section{Discussion}

MIRABEL: In your simulations with what frequency are multiple systems form?

OMUKAI: In the simulation in my talk, we limited the calculation to the axisymmetric case and so we cannot discuss the multiplicity. In other calculations with Machida et al. (2008), we found that with some rotation, sufficiently flat disks are formed during the prestellar collapse, which eventually fragment into binary or multiple systems. Due to the difference in temperature evolution, with the same amount of initial angular momentum, binary formation appears easier in the primordial gas. 